\title{
Keita Kinjo and Shinya Sugawara* Predicting Empirical Patterns in Viewing Japanese TV Dramas Using Case-Based Decision Theory
}

DOI 10.1515/bejte-2015-0014

Published online June 1, 2016

\begin{abstract}
This article empirically analyzes consumer behavior of viewing TV dramas using case-based decision theory. The theory addresses an economic situation with structural ignorance, where states of the world are not naturally given nor simply formulated for a decision-maker. Under this theory, consumers make decisions based on subjective evaluations of previous purchases for similar goods. Our empirical analysis is concerned with viewing decisions on getsuku, the Japanese TV dramas broadcast at 9 pm Monday by the Fuji Television Network. The regularity of the schedule and the long-sustaining popularity of the program enable us to easily collect consumer data. Then, we conduct a web survey of individual audiences on subjective evaluations of previously watched dramas. For our empirical analysis, we utilize a simple linear model of the casebased model that allows the incorporation of flexible inference techniques. Our results demonstrate better performance of the case-based models than models based on traditional expected utility theory regarding both statistical model selection and one-step-ahead prediction. We also reveal that the successful performance of the case-based model in our analysis depends on the availability of individual subjective evaluations and that it is difficult to replace the individual-specific information using demographic information and aggregate data.
\end{abstract}

Keywords: case-based decision models, TV audience rate, Japanese getsuku drama, cultural economics, Kimutaku

JEL Classification: D12, D83, Z11

\section{Introduction}

This article empirically analyzes TV viewing behaviors via a new economic model of case-based decision theory (CBDT). There has been active research

*Corresponding author: Shinya Sugawara, Graduate School of Information Science and Technology, University of Tokyo, Tokyo, Japan, E-mail: sugawara_shinya@ci.i.u-tokyo.ac.jp

Keita Kinjo, Department of Economics, Okinawa International University, Ginowan, Japan 
producing new microeconomic theories regarding consumer behavior under uncertainty that extends the scope of traditional expected utility theory (EUT). However, the validity of most corresponding models has not been checked from an empirical perspective. The present study tries to fill the gap between theoretical and empirical studies for CBDT.

EUT can be applied to decision-making under uncertainly in which states of the world are naturally given, like whether an unopened egg will be fresh or rotten. However, EUT does not work well for uncertainty with structural ignorance (Gilboa and Schmeidler 2001, 45), where states of the world are not naturally given nor simply formulated for a decision-maker. CBDT is a decision theory that addresses structural ignorance. In this theory, consumers predict a value of a good from their past experiences of purchasing similar goods without considering the possible states of the world.

Since it was originally presented by Gilboa and Schemeidler (1995), CBDT literature has been rapidly growing. Theoretical concerns were summarized by Gilboa and Schmeidler (2001) using an axiomatic approach. Gilboa, Lieberman, and Schmeidler (2006) presented the "empirical similarity" model, which is convenient for empirical studies and satisfies the axioms of consumer decisions. Gilboa, Lieberman, and Schmeidler (2006) and Gilboa, Lieberman, and Schmeidler (2011) provide methodologies to handle binary choice variables that are common in empirical analysis. Econometric theories for models for the CBDT are also actively studied by Lieberman (2010), Lieberman (2012), and Lieberman and Phillips (2014).

Along with methodological development, there has been a substantial increase in the number of applied studies of CBDT. Several studies have adopted CBDT for prediction analysis on non-decision problems. Gayer, Gilboa, and Lieberman (2007) applied the empirical similarity model to analyze the process of apartment sale prices and rents using real data. They showed that sale prices are explained better by EUT, while rents fit better to CBDT, which is consistent with the intuition that the speculative nature of the sales market creates a simple evaluation scheme for the goods. Golosnoy and Okhrin (2008) constructed portfolios using real financial return data and showed that portfolios based on CBDT have better performance than those based on EUT. Teitelbaum (2015) analyzed legal reasoning concerning rewards for maritime salvage using CBDT. These studies reported appropriate CBDT performance, which demonstrates the potential explanation powers of the theory in empirical research.

For decision problems, several researchers have provided experimental studies using CBDT and showed good performance. Ossadnik, Wilmsmann, and Niemann (2013) compare CBDT and decision processes without structural ignorance using decision data from a lab experiment. Pape and Kurtz (2013) 
created a software for choice algorithms on CBDT and compared the performance to a widely used psychology algorithm of human classification learning using benchmark data on human categorization. This software was also applied by Guilfoos and Pape (2015) to lab experiment data for the repeated prisoner's dilemma in comparison with a previous method using a probit estimation for cooperation with game structure characteristics by Camera and Casari (2009).

In our empirical application of CBDT, we concentrate on viewing decisions regarding Japanese TV dramas that were broadcast at 9 pm Monday by the Fuji Television Network, often called getsuku (short for "9 pm Monday" in Japanese). The content of TV dramas contains uncertainty, because no drama is completely equivalent to any other. Even after viewing, it is difficult to describe a drama program quantitatively. In the other words, the states of the world concerning TV dramas are not obvious. Furthermore, many dramas aim to surprise viewers, which can be interpreted as that the TV producers often attempt to enlarge the space of states. In such a circumstance, viewing decisions on TV dramas along with consumption of other cultural goods are likely to have structural ignorance.

To explain the potential advantage of CBDT in this context, we present a brief history of the theme of mystery in getsuku. From the 1980s, getsuku became popular for its several successful love stories, whereas mystery was such a minor theme that there had been only three dramas produced from 2000 to the third quarter of 2007. In the fourth quarter of 2007, however, Galileo, a mystery drama based on short stories by Keigo Higashino ${ }^{1}$ was produced. This drama obtained a high audience rate (exceeding $20 \%$ of Japanese viewers), surpassing the audience levels of the eight preceding getsuku dramas. After the success of Galileo, there have been six mystery dramas produced from 2008 to the second quarter of 2013. Such succeeding production of mystery dramas can be naturally explained by a response of TV producers to consumers driven by CBDT.

The main contribution of our study is a unique data construction of real consumer behaviors via a web survey. In applying CBDT to real consumption data, it is difficult to quantify subjective evaluations of previous purchases. Our study overcomes this difficulty using direct measurement with a web survey. The web survey can be used to easily collect retrospective information as well as

1 Several novels that share the protagonist with Galileo were translated into English. Higashino (2012), in particular, was nominated for an Edgar Allan Poe Award for the best mystery novel in 2012. 
demographic characteristics of individuals, and it has become easier to implement. Since the late 1980s, getsuku dramas have received high audience rates (between 10 to $35 \%$ of viewership) and have become the best-known youthoriented dramas in Japan. Because of the regularity of its schedule and longsustained popularity, it is easier to collect consumer data for viewing getsuku dramas than for most other cultural goods. Although such a retrospective survey is likely to have a recollection bias, our data might avoid this problem owing to the regularity of the schedule and the long-sustained popularity of getsuku. As a result, we obtained a panel dataset that records individual viewing behavior for multiple dramas.

As a resource for econometric analysis, web surveys have a potential weakness in the form of sample selection, as summarized by Bethlehem (2010). Although our study is also exposed to the risk of sample selection, we have three responses to such criticism. First, we know of no natural reason why those who make a case-based decision should be more or less likely to participate in web surveys. Thus, sample selection, if any, does not interfere with our main research topic, which is empirical validation of CBDT. Second, viewing behavior of TV dramas might be a topic that is less affected by sample selection than other topics of economic study, because viewing TV and internet surfing are both indoor leisure activities. Furthermore, internet access coverage in Japan reached 79.1\% in 2011(Japan Ministry of Public Management, Home Affairs, and Posts and Telecommunications 2012). Therefore, the coverage of potential web survey participants is likely to overlap that of potential drama audiences. Third, it is difficult to obtain unbiased survey data that contain individual subjective evaluations for past TV programs. Under the data availability problem, a web survey is perhaps the best practical resource.

In our econometric analysis for CBDT, we do not utilize the empirical similarity model but rather construct a simple linear probit-type model on viewing behaviors. To obtain detailed properties of the model in empirical situations, we construct a homogeneous case-based model without demographic information and a heterogeneous case-based model with demographic information. Unlike experimental data, real data often have uncontrolled characteristics. Our heterogeneous model methodology, in which the similarity functions depend on observable consumer characteristics, can stimulate flexible empirical applications of CBDT.

To validate CBDT performance, we also estimate conventional probit models based on EUT, in which individual characteristics, drama characteristics, and subjective evaluations are included as explanatory variables. We compare the performance of models regarding statistical model selection and one-step-ahead prediction for viewing behavior. 
We further present methods for out-of-sample prediction analysis. As summarized in Danaher, Dagger, and Smith (2011), TV advertisements are highly affected by program audience rate. Therefore, if possible, out-of-sample prediction for viewing behavior among the general population is of practical importance. However, naive out-of-sample prediction based on CBDT requires individual subjective evaluations for the prediction sample, which are burdensome to collect. As a feasible approach to out-of-sample prediction, we also propose a method that utilizes average subjective evaluations among those sharing demographic characteristics.

Our empirical analysis yields the following results. Based on statistical model selection and one-step-ahead prediction, CBDT clearly outperforms EUT. For CBDT, specifications with and without demographic characteristics work similarly in both model selection and one-step-ahead prediction. This result shows that CBDT works well even without consumer characteristics other than individual subjective evaluations for our data. Furthermore, outof-sample prediction using average information shows poor performance, whereas naive prediction shows good performance. These results imply that the aggregate information cannot replace the individual subjective evaluations in CBDT.

Our simple linear model for CBDT can adopt flexible extensions for empirical applications on general consumer behavior. As examples of important extensions, we introduce the premium for recently viewed dramas and effects of the published audience rate to analyze peer effects. Our estimation results show that these extensions improve the estimation performance.

With respect to economic literature other than CBDT, there are both theoretical and empirical studies that address the relationship between current and previous decisions. In economic theory, the rational addiction model of Becker and Murphy (1988), in which utility can be changed through accumulation of purchase experiences, can be applied to situations similar to those of CBDT. A learning-by-consuming model, which was used by Lévy-Garbova and Montmarquette (1996) in analysis regarding theatrical performances in France using subjective evaluations, has a motivation similar to that of CBDT. Thus, we can say that CBDT is a method for providing a micro-foundation explicitly to the learning-by-consuming behavior model. Brito and Barros (2005) and Seaman (2006) provide more discussions of the theoretical issues and related empirical methodologies in the context of cultural economics.

For empirical studies, interdependence among decision history has been investigated in state-dependent models. The state-dependent model suggests that consumers tend to purchase the same good repeatedly. Moshkin and Shachar (2002) and Kinjo and Ebina (2015) applied such a model to an empirical 
analysis of TV viewing behavior. From an empirical perspective, CBDT is more general than an ordinary state-dependent model because CBDT adopts not only previous purchase behavior regarding a specific product but also subjective evaluations for previous purchases in general.

The remainder of this article is organized as follows. In Section 2, we provide a detailed description of the data. Section 3 presents our econometric framework, and the empirical findings are shown in Section 4. Section 5 concludes.

\section{Data}

Two kinds of data are required for the empirical analysis of our CBDT models. The first is the drama characteristics, and the second is the individual consumer characteristics. This section first describes the drama characteristics with a brief explanation of getsuku dramas. We then proceed to describe the consumer characteristics. We also report the design of our web survey.

\subsection{Characteristics of Getsuku Dramas}

A getsuku drama is broadcast by the Fuji Television Network from 21:00 to 21:54 every Monday for 3 months. The program started in the late 1980s. Since showing several successful dramas in the early 1990s such as Tokyo Love Story and The 101st Marriage Proposal (101-kai me no puropozu), the program has become the most popular youth-oriented series of dramas in Japan. Getsuku dramas have a large influence on popular culture not only in Japan but also in other Asian countries, as discussed in Iwabuchi (2004).

Although a person's lifetime viewing experience is referred to in CBDT, it is quite burdensome to collect such retrospective information in empirical studies. Therefore, we limit our attention to only $T=54$ getsuku drama series from January 2000 to April 2013.

To define the similarities between dramas in CBDT, we adopt the following $K=6$ drama characteristics and designate $\mathbf{x}^{P}$ as the vector of associated variables. The first category of characteristics is the drama themes. As candidate themes, we consider the three themes of comedy, love story, and mystery, denoted as Comedy, Love, and Mystery, respectively, where multiple themes are allowed for each drama. Because there is no official categorization of themes, we subjectively determine the themes of the dramas based on the information available on the homepages for each drama. The second category concerns the type of original 
work developed for the drama. This category of variables might represent the synergy effects of multiple media. Specifically, we introduce a novel origin dummy (Novel). ${ }^{2}$

The third category of drama characteristics is the cast, whose large influence on TV viewing behavior was shown in Shachar and Emerson (2000). To consider cast effects, we first determined the three main characters of the drama using its homepage. We then consider whether influential actors are cast for these characters. We investigate casting effects for two categories. The first category is actors who are managed by Johnny \& Associates, Johnny's Jimusho in Japanese and denoted by Johnny's. The second category is the actor Takuya Kimura, denoted using his nickname Kimutaku. Johnny \& Associates is an acclaimed management agency for groups of young male pop singers. Many members of these groups are also popular actors and are often cast in getsuku. Takuya Kimura is a famous actor who was cast in seven getsuku dramas during our research period. Takuya Kimuya is also managed by Johnny \& Associates as a member of the best-known male group in Japan, SMAP, but we do not include him in the construction of the Johnny's dummy. Furthermore, we do not adopt a cast variable that includes actresses, because there were no actresses who appeared as a main character in more than three getsuku dramas during our research period.

In the Section 4.3 for extended models, we introduce the published audience rate for dramas as an additional explanatory variable. The regular audience rate of all TV programs is researched and published by Video Research Ltd. in Japan. ${ }^{3}$ Video Research conducts a household sample survey over each minute, but we utilize the overall average during the getsuku broadcast times.

\subsection{Individual Characteristics}

We conducted a web survey of individual viewers through Macromill Inc. on August 22nd and 23rd, 2013. Their monitor pool population consisted of

2 Another candidate origin is comics (manga), which are popular in Japan, but there only three getsuku dramas based on comics were broadcast during the our research period. Thus, we do not utilize this as an origin type.

3 The Japanese national TV network Nihon Housou Kyoukai also researches audience rates, but it covers only 5 weeks per year. The Nielsen Company once operated in Japan before the launch of Video Research but withdrew from Japan in 2000. Fujihira (2007) presents more details about audience rates in Japan. 
$1,147,370$ individuals on August 1st, $2013 .{ }^{4}$ From this population, we utilize stratified sampling for gender and age by 10-year group based on the 2010 Census. We restrict our sample to individuals who live alone in order to remove peer effects from family members as analyzed by Yang, Narayan, and Assael (2006). We also restrict our sample to only those who are 20 to 69 years old at the time of the web survey.

The dependent variable is a viewing dummy for each getsuku drama that takes a value of 1 when an individual viewed the drama at least once during its season. Audience members may view only a few episodes. Such viewing behavior can inform our research because the behavior might be associated with low subjective evaluation of the drama. Thus, we include such behavior in the definition of our viewing dummy. ${ }^{5}$ To maintain the sequential order in accumulation of viewing experience, we ask only real-time viewing behavior and do not count viewing conducted after the formal broadcasting period. On the other hand, we allow viewing via the internet or recorded video tapes if it occurs during the same period as the broadcast.

An important component for our model is subjective evaluation for viewed dramas. Because our web survey is a one-shot survey, this information is interviewed using retrospective questions. We asked for a subjective evaluation as a discrete variable that can be chosen from 11 levels. In our web survey, the subjective evaluations are asked by retrospective questions about dramas broadcasted at most 13 years ago. To make a consistent empirical analysis, we assume that evaluations are unchanged at the times of the decision making and of the interview.

In our empirical analysis, we also utilize three demographic characteristics of individuals: gender, age, and education level. For education, we do not ask previous status at the time each drama is viewed to minimize the interview burden. Thus, we need to assume that these demographic characteristics are time-invariant. The implicit assumption of time-invariance is also assigned for the design of stratified sampling in the sense that single people at the time of the survey were assumed to be single in the retrospective survey periods. For age, because we do not ask birthday but only ask age at the time of survey, age at the time of the drama broadcast is obtained by subtracting (2013 minus the year of broadcasting) from the age specified in 2013.

\footnotetext{
4 This number was taken from http:www.macromill.commonitor_infopdf20130801web.pdf, accessed on May 9th, 2014

5 An interesting suggestion by an anonymous referee is to use the number of viewed episodes as a continuous outcome variable. Unfortunately, we cannot adopt this approach because our web survey did not collect such information.
} 
As mentioned in the Section 3.1 for model description, we need to discretize all demographic characteristics in our CBDT model. Gender takes two values, Male and Female. Age is decomposed into two statuses: 39 years old or less (Young) and 40 years old or more (0ld). For education level, we utilize two categories: university graduate or more (University) and less than university graduate (High School). Consequently, we have $2^{3}=8$ categories of demographic characteristics, which we denote as $\mathbf{x}^{I}$.

\subsection{Descriptive Statistics}

Table 1 reports the descriptive statistics. Using our data collection scheme, we obtain data for $N=415$ individuals and $T=54$ dramas, and their multiplication is $N T=22$, 410. We also summarize information about the number of dramas viewed by individuals in Table 1 . As shown, approximately $1 / 4$ of the individuals did not view getsuku dramas, ${ }^{6}$ whereas less than $5 \%$ of the individuals viewed 40 or more of the 54 dramas.

The subjective evaluation takes a value of zero for non-viewed dramas, whereas it takes eleven values among $\{0.1,0.2, \ldots, 1.1\}$ for viewed dramas. In demographic characteristics, Young reflects the age status at the time of our web survey. We also report the fractions of individuals who comprise each of the eight categories of discretized demographic characteristics $\mathbf{x}^{I}$. As shown in the table, all categories have sufficient numbers of individuals.

\section{An Empirical Model for TV Viewing CBDT}

\subsection{Model Description}

We utilize a panel dataset of individuals who choose whether to view a TV drama or not. The dataset consists of $N$ individuals who are indexed as $i=1,2, \ldots, N$ and $T$ dramas that are sequentially ordered via the time index $t=1,2, \ldots, T$. To analyze viewing behavior for the $t$ th drama, the dependent variable, $y_{i t}$, represents a viewing decision dummy that takes a value of 1 when the $i$ th consumer views the $t$ th drama. Let $\mathbf{x}_{t}^{P}$ be a $K \times 1$ vector of drama characteristics that are observable both by

\footnotetext{
6 The number of those who did not watch any getsuku drama seems large to apply the CBDT. To check the robustness of our methodology, we employ estimation of the CBDT models using only the subsample of individuals who have viewed at least a getsuku drama. The estimation results are generally compatible to the results from the full sample.
} 
Table 1: Descriptive statistics. F, M, Y, O, U and H are short for Female, Male, Young, Old, University and High school, respectively.

\begin{tabular}{|c|c|c|c|}
\hline \multicolumn{2}{|l|}{ Descriptive statistics } & \multirow{2}{*}{$\begin{array}{c}\text { Mean } \\
10.45\end{array}$} & \multirow{2}{*}{$\frac{\text { S.D. }}{(11.99)}$} \\
\hline Individual & \#Dramas Viewed by an individual & & \\
\hline \multirow[t]{6}{*}{ characteristics } & $\begin{array}{l}\text { Subjective evaluation } \\
v \in\{0,0.1, \ldots, 1.1\}\end{array}$ & 0.16 & $(0.34)$ \\
\hline & $\begin{array}{l}\text { Subjective evaluation of a viewer } v \\
\text { given } y=1\end{array}$ & 0.81 & $(0.23)$ \\
\hline & Female & 0.4 & $(0.49)$ \\
\hline & Young & 0.47 & $(0.50)$ \\
\hline & University & 0.48 & $(0.50)$ \\
\hline & Observations & & $N=415$ \\
\hline \multirow{8}{*}{$\begin{array}{l}\text { Drama } \\
\text { characteristics }\end{array}$} & Novel & 0.13 & $(0.34)$ \\
\hline & Johnny’s & 0.33 & $(0.48)$ \\
\hline & Kimutaku & 0.13 & $(0.34)$ \\
\hline & Comedy & 0.28 & $(0.45)$ \\
\hline & Love & 0.57 & $(0.50)$ \\
\hline & Mystery & 0.19 & $(0.39)$ \\
\hline & Published audience rate & 17.01 & $(4.31)$ \\
\hline & Observations & & $T=54$ \\
\hline \multirow{2}{*}{$\begin{array}{l}\text { Summary statistics for viewing } \\
\text { behaviors }\end{array}$} & Drama viewing dummy $y$ & 0.19 & $(0.40)$ \\
\hline & Observations & & $N T=22410$ \\
\hline \multicolumn{2}{|l|}{ Count for individuals } & Count & \\
\hline \multirow[t]{6}{*}{ \#Viewed dramas } & $\#$ Viewed drama $=0$ & 94 & \\
\hline & $0<\#$ Viewed drama $\leq 10$ & 175 & \\
\hline & $10<\#$ Viewed drama $\leq 20$ & 66 & \\
\hline & $20<\#$ Viewed drama $\leq 30$ & 48 & \\
\hline & $30<\#$ Viewed drama $\leq 40$ & 16.00 & \\
\hline & $40<\#$ Viewed drama & 16.00 & \\
\hline \multirow[t]{8}{*}{ Individual characteristics } & $\mathrm{F}, \mathrm{Y}, \mathrm{H}$ & 39 & \\
\hline & $\mathrm{F}, \mathrm{Y}, \mathrm{U}$ & 36 & \\
\hline & $\mathrm{F}, \mathrm{O}, \mathrm{H}$ & 69 & \\
\hline & $F, 0, U$ & 22 & \\
\hline & $M, Y, H$ & 54 & \\
\hline & $M, Y, U$ & 66 & \\
\hline & $\mathrm{M}, \mathrm{O}, \mathrm{H}$ & 53 & \\
\hline & $M, 0, U$ & 76 & \\
\hline
\end{tabular}

consumers and researchers. We assume that the viewing decision is made when the consumer's subjective prediction for his or her own latent utility, $y_{i t}^{\star}$, is nonnegative.

The subjectively predicted utility $y_{i t}^{\star}$ is a function of a difference of the subjective evaluation for previously viewed dramas, denoted by $v_{i \tau}$ (where $\tau=1, \ldots, t-1$ ) 
and the aspiration level $h_{i t}$. For the subjective evaluation, we define $v_{i \tau}=0$ for dramas that are not viewed. For viewed dramas, we define $v_{i \tau} \in[0.1,0.2, \ldots, 1.1]$, where a larger number corresponds to higher satisfaction. The aspiration level, which is discussed in detail by Gilboa and Schmeidler (1996), is a reference value of the utility beyond which the decision-maker does not try alternatives.

In decision-making, the difference $v_{i \tau}-h_{i t}$ is weighted by similarity in drama characteristics between the $\tau$ th and $t$ th dramas. The similarity is measured using a similarity function $s_{i}\left(\mathbf{x}_{t}^{P}, \mathbf{x}_{\tau}^{P}\right)$, which we allow to be individual-specific. The similarity-weighted subjective evaluations for past dramas affect the predicted utility as additively separable terms as well as a constant term with a coefficient $\tilde{\delta}_{i}$ and a mean-zero error term $\tilde{\varepsilon}_{i t}$ that represent unobserved components. In other words,

$$
\begin{aligned}
y_{i t}^{\star} & =\tilde{\delta}_{i}+\sum_{\tau<t} s_{i}\left(\mathbf{x}_{\tau}^{P}, \mathbf{x}_{t}^{P}\right)\left(v_{i \tau}-h_{i t}\right)+\tilde{\epsilon}_{i t}, \\
y_{i t} & =I\left[y_{i t}^{\star} \geq 0\right],
\end{aligned}
$$

where the indicator function $I[S]$ takes a value of unity if $S$ holds.

For the similarity function, previous studies commonly adopted a function based on the Euclidean norm for characteristics between dramas. This choice of the similarity function does not fit our data, because we choose only dummy variables for drama characteristics. Instead, we first construct an indicator function that takes a value of unity only when the two dramas both have the same characteristics. For example, if two dramas are both based on novels, the indicator function regarding Novel takes a value of unity. Furthermore, we multiply an individual-specific weight for each drama characteristic pattern for individual $i$ on the $k=1, \ldots, K$ th drama characteristics. In other words, our similarity function is

$$
s_{i}\left(\mathbf{x}_{t}^{P}, \mathbf{x}_{\tau}^{P}\right)=\sum_{k=1}^{K} \omega_{i k} I\left[x_{k t}^{P}=x_{k \tau}^{P}=1\right] .
$$

Through calculation, the estimation model generates the following simple linear model.

$$
y_{i t}^{\star}=\delta+\mathbf{x}_{i t}^{\prime} w_{i}+\varepsilon_{i t},
$$

where

$$
\begin{aligned}
\mathbf{x}_{i t} & =\left\{\sum_{\tau<t} I\left[x_{1 t}^{P}=x_{1 \tau}^{P}=1\right] v_{i \tau}, \ldots, \sum_{\tau<t} I\left[x_{K t}^{P}=x_{K \tau}^{P}=1\right] v_{i \tau}\right\}^{\prime} \\
\delta+\varepsilon_{i t} & =\tilde{\delta}_{i}-\sum_{k=1}^{K} \sum_{\tau<t} \omega_{i k} I\left[x_{k t}^{P}=x_{k \tau}^{P}=1\right] h_{i t}+\tilde{\varepsilon}_{i t} \\
w_{i} & =\left(\omega_{i 1}, \ldots, \omega_{i K}\right)^{\prime} .
\end{aligned}
$$


In eq. [2], we define that the expected value with respect to all $i$ and $t$ of the right-hand side is $\delta$ and mean-zero variations for each $i$ and $t$ is $\varepsilon_{i t}$. This equation claims that the terms on the right-hand side, which include the aspiration level, is decomposed into a time-invariant constant term $\delta$ and a variable factor $\varepsilon_{i t}$.

We adopt a parametric assumption for the standard probit model. Specifically, we assume $\varepsilon_{i t}$ to be independent and identically distributed according to the standard normal distribution. Our estimands are the constant term $\delta$ and the $K$ weight terms in the similarity function $\omega_{i k}$, which can be identified using the variation of $v_{i \tau}$. We call $\left(\delta, w_{i}{ }^{\prime}\right)^{\prime}$ as a "coefficient vector." Because $\omega_{i k}, k=1, \ldots, K$ is a weight used to measure similarity, it must take a nonnegative value. In our estimation, we estimate the probit model without constraints and check whether weights are nonnegative using the post-estimation $t$ test. On the other hand, unlike the weight term $\omega_{i k}, \delta$ can take a negative value.

In the above formulation of the empirical CBDT model, we assume that individuals adopt an equivalent model of decision making as in eq. [1] for 13 years. This assumption is required to identify parameters for the CBDT model, but comprises a strong restriction on individual decision making. Then, in Section 4.3, we try to relax this assumption by introducing a time-variant elements into the CBDT model.

To understand more details about CBDT in empirical situations, we adopt two specifications on $w_{i}$. Our first specification is a homogeneous model without demographic information. The homogeneous case-based model does not utilize any individual-specific information other than the subjective evaluations for previously viewed dramas. Thus, we have $w_{i}=w$ for any $i$.

Our second specification is a heterogeneous model with demographic information. In the heterogeneous case-based model, the weight terms of the similarity function are allowed to depend on demographic characteristics of individuals. For simplicity, we do not assume that weights for all drama characteristics vary with all individual characteristics, but adopt the following schemes. First, the weight for Novel does not depend on demographic information, because we do not have an intuition concerning the demographic variation of the effects of this variable. Second, for cast effects of Johnny's and Kimutaku, weight terms can differ in terms of gender because these variables are concerned with actors. Finally, Comedy, Love, and Mystery may depend on all demographic characteristics.

The assumption that $\varepsilon_{i t}$ follows an independent and identical distribution is strong, because $\varepsilon_{i t}$ can include individual and time specific elements from the definition. This assumption is technically required to employ the probit 
analysis. Furthermore, this assumption naturally holds for the model without the aspiration level if we also assume $\tilde{\delta}_{i}=\delta$, because $h_{i t}=0$ for any $i$ and $t$ implies that $\varepsilon_{i t}=\tilde{\varepsilon}_{i t}$. On the other hand, it is difficult to find an intuitive model which guarantees the assumption of $\varepsilon_{i t}$ if the aspiration level is not zero. Because the econometric analysis for the aspiration level is an important task for future researches, we propose three econometric methodologies to loose the assumptions, although they are beyond the scope of this study, as follows.

The first methodology is individual-specific fixed or random effects. In this study, however, introduction of them into our estimation has technical difficulties. Because several individuals did not view any dramas, a multicollinearity problem occurs for estimation of the individual fixed effects. We can employ estimation by eliminating the fixed-effect terms for those individuals, but this operation prevents us from conducting a prediction analysis.

The second methodology is explicit estimation for $h_{i t}$. In our model, the aspiration level $h_{i t}$ is assumed to be a random variable for which we do not construct a statistical model. However, it is also an important question whether we can estimate $h_{i t}$ as a parameter. To consider this question, we simplify a problem to assume the time-invariant aspiration level as $h_{i t}=h_{i}$ for all $i$ and $t$. Then, the term related to $h_{i}$ in eq. [2] becomes

$$
-h_{i} \sum_{k=1}^{K} \omega_{i k} \sum_{\tau<t} I\left[x_{k t}^{P}=x_{k \tau}^{P}=1\right]
$$

As a result, we obtain a random coefficient model where the parameter $h_{i}$ is individual-specific. Because the number of parameters increases along with the sample size, estimation for discrete choice models with random coefficients is not straight-forward and we need econometric techniques such as a nonparametric estimator by Ichimura and Thompson (1998) or a Bayesian estimator by Allenby and Rossi (1998). Furthermore, in our analysis, we have an additional task to decompose $\omega_{i k}$ and $h_{i}$ in the above term. Such an advanced econometric analysis is a beyond the scope of this study.

The third methodology is structural estimation for the aspiration level. As Ossadnik, Wilmsmann, and Niemann (2013)) presented, the aspiration level can be directly estimated with an explicit assumption on its adjustment process. However, as discussed in Gilboa and Schmeidler (1996) and Gilboa and Schmeidler (2001, pp. 39-42), there can be several models on the adjustment process and it is not trivial to choose one model among them. 


\subsection{Framework for Model Comparison with the EUT Models}

\subsubsection{Proposed EUT Models}

To see the empirical validity of CBDT, we compare its performance with several EUT specifications. For this purpose, we carefully construct compatible models for EUT as follows.

First, we construct a standard linear probit model as a proper approximation of the EUT theory for empirical analysis. We consider a viewing decision on a getsuku drama $t$ by an individual $i$, where $\tilde{\mathbf{z}}_{i t}$ is a vector of various elements which can affect this decision. We define $S$ as a set of states of the world. In our context, this is a set of possible getsuku dramas. We assume that the state space is known in EUT, but we actually think this is not a realistic assumption and CBDT is more appropriate in the sense that it works without such an assumption. On each outcome $s \in S$, we let $p\left(s \mid \tilde{\mathbf{z}}_{i t}\right)$ and $u\left(s \mid \mathbf{z}_{i t}\right)$ be a probability measure and an assigned utility, both of which are affected by $\tilde{\mathbf{z}}_{i t}$. The expected utility of watching a getsuku drama is

$$
U_{i t}=\sum_{s \in S} p\left(s \mid \tilde{\mathbf{z}}_{i t}\right) u\left(s \mid \tilde{\mathbf{z}}_{i t}\right)
$$

In practice of empirical analysis, it is difficult to observe all elements which affect the decision making. We consider a situation in which we only have observable factors $\mathbf{z}_{i t}$. Using the first order Taylor expansion, we obtain a linear approximation of the above expected utility as

$$
U_{i t} \simeq \mathbf{z}_{i t} \gamma+\eta_{i t}
$$

where $\eta_{i t}$ is a function of unobservable components in $\tilde{\mathbf{z}}_{i t}$. For identification, we assume that the expected utility of not viewing the drama is standardized to be zero. Then, the resulted decision model is summarized that the drama is viewed if $\mathbf{z}_{i t} \gamma+\eta_{i t} \geq 0$ and otherwise not viewed. This is a standard econometric model for the discrete choice. Furthermore, letting $\eta_{i t}$ follow a standard normal distribution, we obtain the standard linear probit model as a natural approximation of EUT.

Second, we discuss our strategy to compare CBDT and EUT models. Our discussion heavily depends on Gayer, Gilboa, and Lieberman (2007) who compared the CBDT model and the rule-based model, which has a similar role to our EUT as a conventional model. 
For proper empirical comparison of CBDT and EUT, we need to take care of two issues, distinguishability of two approaches and fairness of model comparison. For the distinguishability, there is a difficulty that these two models can be theoretically equivalent without any restriction, as shown by Matsui (2000). Then, in their empirical analysis, Gayer, Gilboa, and Lieberman (2007) made two ways to guarantee the distinguishability. First, they constructed very intuitive, simple parametric forms for the two approaches to make them distinguishable. However, we cannot follow this way because our empirical CBDT and EUT are both based on the equivalent linear probit model.

Another effort by Gayer, Gilboa, and Lieberman (2007) is a distinct choice of explanatory variables. Their rule-based model did not utilize past prices, which play a similar role to past subjective evaluations in our analysis, while their CBDT utilized them. In our analysis, we try to follow this approach to guarantee distinguishability. However, ignorance of whole information from the subjective evaluation might violate fairness of model comparison. Then, we adopt information from subjective evaluations into EUT in a completely different way to CBDT.

Depending on the choice of explanatory variables $\mathbf{z}_{i t}$, we separately consider three alternative models based on EUT. The first model utilizes only the consumer and drama characteristics $\mathbf{x}^{I}$ and $\mathbf{x}^{P}$ and their cross-terms, without subjective evaluations. These variables are standard choice in the marketing analysis of TV viewing such as Rust and Alpert (1984) and Shachar and Emerson (2000). On the choice of cross-terms, we utilize the combinations corresponding to those for the heterogeneous CBDT model. Specifically, we utilize Female times Johnny's and Kimutaku, and all demographic characteristics times the drama themes, Comedy, Love, and Mystery.

The other two EUT models utilize subjective evaluation in addition to individual and drama characteristics. EUT models with subjective evaluations can be justified as a variant of Bayesian decision-making, where subjective evaluation, which might be related to the realized quality of past dramas, is utilized for Bayesian updating of $p\left(s \mid \tilde{\mathbf{z}}_{i t}\right)$ in eq. [3]. One EUT model includes the subjective evaluation only of one previous drama, $v_{t-1}$. This is a model in which older experience is not used in the Bayesian updating. In this model, distinguishability is guaranteed because subjective evaluations for older dramas are not used in EUT. Another model utilizes a mean of subjective evaluations of all previously watched dramas. Inclusion of this variable corresponds to the Bayesian updating for the entire category of getsuku dramas. In this model, previous subjective evaluations are utilized via a functional form which is completely different from that in CBDT. 


\subsubsection{Statistical Methodologies for Model Comparison}

We compare models using model selection statistics and prediction analysis. For model selection statistics, we utilize the Akaike Information Criterion (AIC) and Schwartz's Bayesian Information Criterion (BIC). In addition to these information criteria, we report pseudo $R^{2}$ of McFadden (1974) to show the goodness of fit of models.

For prediction analysis, we conduct three schemes to predict the choice probabilities of viewing dramas. The first prediction scheme is one-step-ahead prediction. This prediction scheme separates dramas into two groups of samples for estimation and for prediction, and it utilizes all individuals for both estimation and prediction. We adopt the first $T_{e}$ dramas for the estimation sample and the $\left(T_{e}+1\right)$ th drama for the prediction sample. We separately conduct four prediction analyses for each of $T_{e}+1=51,52,53,54$.

The remaining two prediction schemes are out-of-sample methods. Out-ofsample prediction separates individuals into estimation and prediction samples, whereas it utilizes all dramas for both estimation and prediction. The second prediction scheme utilizes a naive approach in which subjective evaluations for prediction samples are assumed to be available in prediction. Then, this naive out-of-sample prediction is incorporated simply by plugging in coefficient estimates for prediction criterion.

However, in a realistic situation, subjective evaluations are not available for prediction samples. For example, if prediction is conducted for audience rate among the general population, it is impossible to obtain such individuallevel information for the enormous prediction sample. Then, in the third prediction scheme, we utilize the aggregate data among the estimation sample to mimic individual subjective evaluations. Specifically, we utilize the mean of subjective evaluations of individuals in the estimation sample for each drama. For the model with demographic information, we take the mean among individuals in the estimation sample who share the demographic characteristics $\mathbf{x}^{I}$ depending on the drama characteristics. Specifically, we utilize the average of all individuals for Novel, gender-specific averages for Johnny's and Kimutaku, and averages for all demographic characteristics for Comedy, Love, and Mystery. Let $\bar{v}_{i \tau, k}^{E}$ be the average subjective evaluation that corresponds to the $k$ th drama characteristics. Given the coefficient estimates $\hat{w}_{i}$, the predicted choice probabilities for drama $t$ and individual $i$ who belong to the prediction sample are

$$
\operatorname{Pr}\left(\widehat{y_{i t}=1}\right)=\Phi\left(\hat{\delta}+\tilde{\mathbf{x}}_{i t, P}^{\prime} \hat{\omega}_{i}\right),
$$


where

$$
\tilde{\mathbf{x}}_{i t, P}=\left\{\sum_{\tau<t} I\left[x_{1 t}^{P}=x_{1 \tau}^{P}=1\right] \bar{v}_{i \tau, 1}^{E}, \ldots, \sum_{\tau<t} I\left[x_{K t}^{P}=x_{K \tau}^{P}=1\right] \bar{v}_{i \tau, K}^{E}\right\}^{\prime} .
$$

For out-of-sample prediction with aggregate data on the EUT models with subjective evaluations, we incorporate mean subjective evaluations of the estimation sample.

\subsection{Distinct Properties of Our CBDT Models}

Our CBDT models have the following differences compared to previous empirical studies. First, we do not utilize the empirical similarity model of Gilboa, Lieberman, and Schmeidler (2006), in which latent utility takes the following form.

$$
y_{i t}^{\star}=\frac{\sum_{\tau<t} s\left(\mathbf{x}_{t}^{P}, \mathbf{x}_{\tau}^{P}\right) v_{i \tau}}{\sum_{\tau<t} s\left(\mathbf{x}_{t}^{P}, \mathbf{x}_{\tau}^{P}\right)}+\varepsilon_{i t} .
$$

Instead, we utilize a simple linear probit CBDT model that allows the incorporation of flexible inference techniques. ${ }^{7}$ To show the validity of our methodology, we conduct several additional inferences into our empirical analysis in later sections. These extensions provide useful implications for analyzing general consumer behavior.

Second, in our analysis, the determination of $v_{i t}$ is not explicitly modeled in our model setup. This is a clear distinction of our CBDT model to the EUT model. In the EUT model, $v_{i t}$ and the latent utility are directly related. However, in the CBDT model, predicted and realized utilities are not required to be equivalent.

\section{Empirical Results}

\subsection{Estimation Results}

Tables 2 and 3 report marginal effects from the probit estimation results for all models with the model selection criterion. Models (1) to (5) represent

7 We also tried estimation with the empirical similarity model, but our estimation for the specification with demographic information yielded large standard errors for estimators so we do not utilize this model in our empirical analysis. The sample size of our data seems insufficient for sharp identification for the complicated functional forms of the empirical similarity model. 
Table 2: Marginal effects of probit model for the CBDT.

\begin{tabular}{|c|c|c|c|c|c|}
\hline \multicolumn{6}{|c|}{ Dependent variable: Drama Viewing Dummy } \\
\hline \multirow{2}{*}{$\frac{\text { Drama }}{\text { Novel }}$} & \multirow{2}{*}{$\begin{array}{l}\text { Demographic } \\
\text { All }\end{array}$} & \multicolumn{3}{|c|}{ (1) } & \multirow{2}{*}{$\frac{(2)}{(0.010)}$} \\
\hline & & $0.067^{\star \star \star}$ & $(0.010)$ & $0.068^{\star \star \star}$ & \\
\hline Johnny's & All & $0.003^{*}$ & $(0.002)$ & & \\
\hline Johnny's & Female & & & 0.002 & $(0.003)$ \\
\hline Johnny's & Male & & & 0.004 & $(0.003)$ \\
\hline Kimutaku & All & $0.088^{\star \star \star}$ & $(0.005)$ & & \\
\hline Kimutaku & Female & & & $0.084^{\star \star \star}$ & $(0.007)$ \\
\hline Kimutaku & Male & & & $0.091^{\star * \star}$ & $(0.007)$ \\
\hline Comedy & All & $0.046^{\star \star \star}$ & $(0.002)$ & & \\
\hline Comedy & $\mathrm{F}, \mathrm{Y}, \mathrm{H}$ & & & $0.050^{\star \star \star}$ & $(0.004)$ \\
\hline Comedy & $F, Y, U$ & & & $0.046^{\star \star \star}$ & $(0.004)$ \\
\hline Comedy & $\mathrm{F}, \mathrm{O}, \mathrm{H}$ & & & $0.035^{\star \star \star}$ & $(0.006)$ \\
\hline Comedy & $\mathrm{F}, \mathrm{O}, \mathrm{U}$ & & & $0.035^{\star \star \star}$ & $(0.009)$ \\
\hline Comedy & $M, Y, H$ & & & $0.044^{\star \star \star}$ & $(0.005)$ \\
\hline Comedy & $M, Y, U$ & & & $0.045^{\star \star \star}$ & $(0.005)$ \\
\hline Comedy & $\mathrm{M}, \mathrm{O}, \mathrm{H}$ & & & $0.052^{\star \star \star}$ & $(0.009)$ \\
\hline Comedy & $M, O, U$ & & & $0.064^{\star \star \star}$ & $(0.007)$ \\
\hline Love & All & $0.030 * \star \star$ & $(0.001)$ & & \\
\hline Love & $F, Y, H$ & & & $0.032^{\star \star \star}$ & $(0.002)$ \\
\hline Love & $F, Y, U$ & & & $0.034^{\star \star \star}$ & $(0.002)$ \\
\hline Love & $\mathrm{F}, \mathrm{O}, \mathrm{H}$ & & & $0.030^{\star * \star}$ & $(0.003)$ \\
\hline Love & $\mathrm{F}, \mathrm{O}, \mathrm{U}$ & & & $0.019^{\star \star \star}$ & $(0.004)$ \\
\hline Love & $M, Y, H$ & & & $0.026^{\star \star \star}$ & $(0.002)$ \\
\hline Love & $M, Y, U$ & & & $0.025^{\star \star \star}$ & $(0.002)$ \\
\hline Love & $\mathrm{M}, \mathrm{O}, \mathrm{H}$ & & & $0.038^{\star \star \star}$ & $(0.004)$ \\
\hline Love & $M, O, U$ & & & $0.032^{\star \star \star}$ & $(0.003)$ \\
\hline Mystery & All & $0.088^{\star \star \star}$ & $(0.005)$ & & \\
\hline Mystery & $\mathrm{F}, \mathrm{Y}, \mathrm{H}$ & & & $0.091^{\star \star \star}$ & $(0.010)$ \\
\hline Mystery & $F, Y, U$ & & & $0.099^{\star * *}$ & $(0.009)$ \\
\hline Mystery & $\mathrm{F}, \mathrm{O}, \mathrm{H}$ & & & $0.083^{\star \star \star}$ & $(0.009)$ \\
\hline Mystery & $\mathrm{F}, \mathrm{O}, \mathrm{U}$ & & & $0.074^{\star \star \star}$ & $(0.016)$ \\
\hline Mystery & $M, Y, H$ & & & $0.093^{\star \star \star}$ & $(0.010)$ \\
\hline Mystery & $M, Y, U$ & & & $0.090^{\star * *}$ & $(0.009)$ \\
\hline Mystery & $M, O, H$ & & & $0.089^{\star \star \star}$ & $(0.013)$ \\
\hline Mystery & $M, O, U$ & & & $0.077^{\star \star \star}$ & $(0.009)$ \\
\hline Sample si & & 22,410 & & 22,410 & \\
\hline AIC & & 18751 & & 18746 & \\
\hline $\mathrm{BIC}$ & & 18767 & & 18816 & \\
\hline pseudo $R^{2}$ & & 0.149 & & 0.151 & \\
\hline
\end{tabular}

Note: (1) and (2) represent the homogeneous and heterogeneous models, respectively. F, M, Y, $\mathrm{O}, \mathrm{U}$, and $\mathrm{H}$ are short for Female, Male, Young, Old, University, and High school, respectively. Standard errors in parentheses. ${ }^{* \star *},{ }^{* *}$, and ${ }^{*}$ denote $p<0.01, p<0.05$, and $p<0.1$, respectively. 


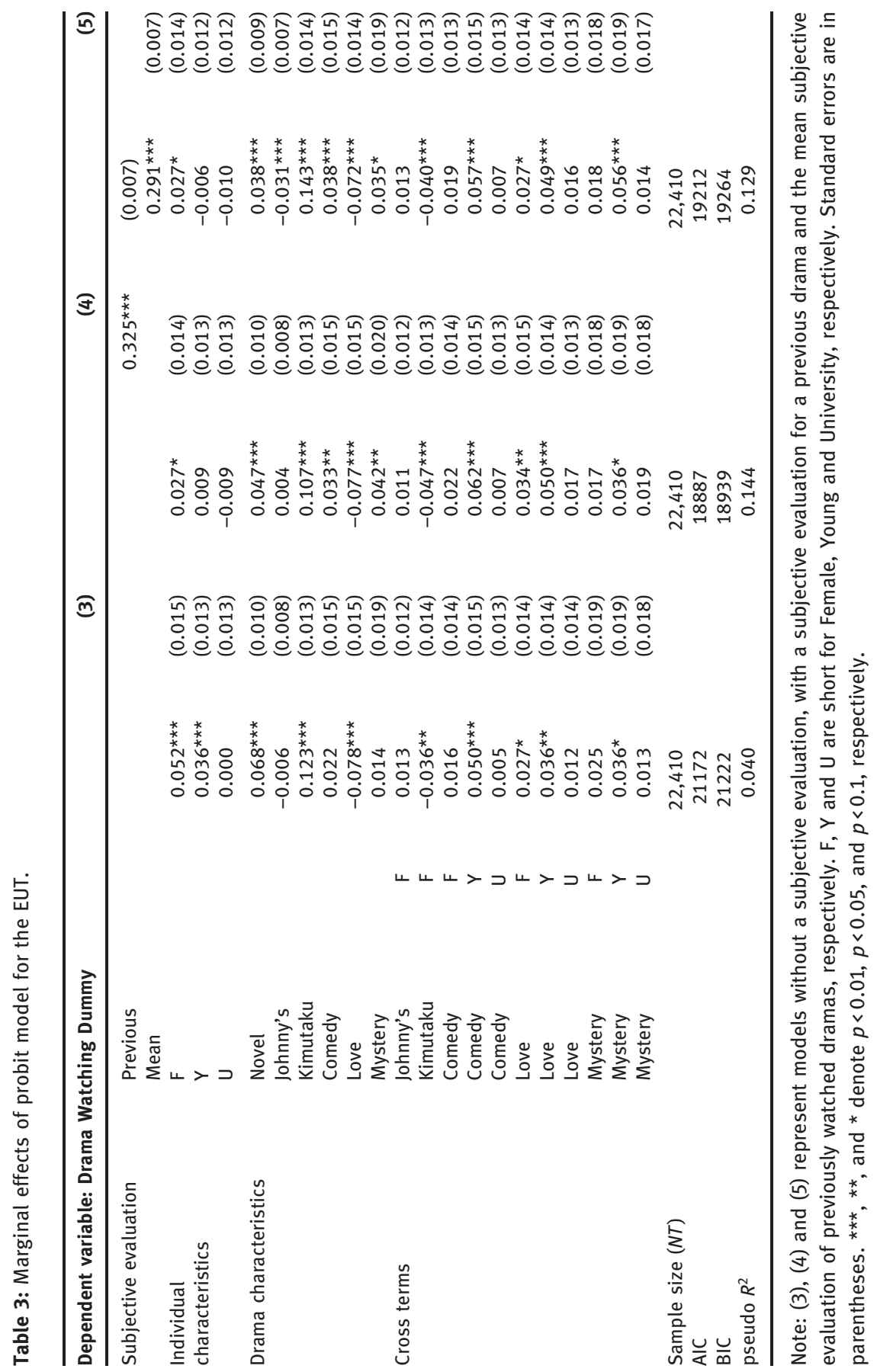


homogeneous and heterogeneous case-based models, EUT models without subjective evaluation, those with subjective evaluation for a previous drama, and the mean subjective evaluation of all previously watched dramas, respectively.

For statistical model selection, the CBDT models outperform the EUT ones for both information criteria and the pseudo $R^{2}$. This result clearly shows the validity of CBDT for TV viewing behavior.

The model selection can also provide a comparison within models for CBDT and EUT. Among the CBDT models, AIC chooses the heterogeneous model, whereas BIC chooses the homogeneous model. This may result from the difference in penalty terms for the definitions of AIC and BIC, that is, the BIC prefers a simpler model. ${ }^{8}$ In short, it might imply that these two specifications do not substantially differ as statistical models and that the CBDT model is a powerful empirical model even without demographic information for our data. Among EUT models, the model selection results show that models with subjective evaluation are preferred to that without it. It also implies that subjective evaluation is a non-negligible factor for viewing decisions and that models using them more intensively like those of CBDT are likely more accurate.

On the goodness of fit among the CBDT models, the pseudo $R^{2}$ for homogeneous and heterogeneous models are 0.149 and 0.151 , respectively. McFadden (1979) stated that this statistics generally shows a lower value than the standard $R^{2}$ and $0.2-0.4$ can be seen as an evidence of excellent fit. Thus, our CBDT models show moderate fit to data in terms of this statistics.

From our coefficient estimates, we have the following implications. First, all weights in CBDT coefficients have positive estimates and are significant except for the coefficients of Johnny's in the model with demographic information. These results guarantee our requirement that weights take nonnegative values and support evidence of the validity of CBDT in viewing decisions for getsuku dramas.

On the other hand, we obtain significantly negative estimates for the constant terms in CBDT, although we do not report them in the table because marginal effects for them are not defined. Specifically, their estimates and standard errors are $-1.257(0.013)$ and $-1.258(0.013)$ for the homogeneous and heterogeneous CBDT models, respectively. This finding provides a reason why our analysis can be compatible to the low observed percentage of dramas viewing, even if all $v_{i t}$ and $\omega_{i k}$ are positive.

Second, the homogeneous and heterogeneous CBDT models in (1) and (2) in Table 2 present similar estimates. We also see in (2) that there is no large difference among demographic characteristic coefficient estimates for each drama characteristic pattern. These results seem striking in comparison to the

8 A formal discussion comparing AIC and BIC is given in Burnham and Anderson (2002) 
EUT estimates. Table 3 shows that in all models based on EUT, several crossterms have significant coefficient estimates, such as Kimutaku times Female, Comedy times Young, and Love times Young.

To explain this finding, we provide the hypothesis that people grasp similarities of drama characteristics in a similar manner regardless of individual characteristics, while subjective evaluations incorporate the difference of individual characteristics into the CBDT. To validate this hypothesis, Table 4 summarizes the means of the subjective evaluations under the selected drama and individual characteristics that correspond to the three significant cross-terms in EUT. For each drama characteristic, we indeed find differences in the mean subjective evaluations, although they are not significant with respect to the individual characteristics.

Table 4: Mean subjective evaluations for selected combinations of drama and individual characteristics.

\begin{tabular}{lrrrr}
\hline & \multicolumn{4}{c}{ Mean subjective evaluation } \\
\cline { 2 - 5 } & \multicolumn{4}{c}{ (A) } \\
\hline Kimutaku (A)Female vs (B)Male & 0.261 & $(0.375)$ & 0.213 & $(0.412)$ \\
Comedy (A)Young vs (B)Old & 0.204 & $(0.313)$ & 0.130 & $(0.372)$ \\
Love (A)Young vs (B)Old & 0.146 & $(0.265)$ & 0.092 & $(0.324)$ \\
\hline
\end{tabular}

Note: Standard deviations are in parentheses.

We cannot claim that this hypothesis holds for general situations, because this is based on only one empirical study. However, it may imply that we do not need to conduct costly collection of individual demographic information for empirical CBDT analysis if we have access to subjective evaluations. Considering the importance of this claim, further empirical studies are required.

For coefficient estimates of each drama characteristic in the CBDT model, Novel has a significantly positive weight. Dramas in this category are generally based on bestsellers, and their adaptation might be recognized as a similar characteristic. For cast variables, we find a large positive weight of Kimutaku and a small weight of Johnny's. Thus, people may identify Takuya Kimura as a more remarkable character for getsuku dramas than any of the other actors who are managed by Johnny \& Associates.

For drama themes, although the difference is small, Mystery has a larger weight for young audience members than for older members in every gender and education group. This result implies that the recent popularity of mystery dramas, which was mentioned earlier, might be owing to recognition by young audience members. On the other hand, we do not find a particular demographic pattern for weights on Comedy or Love. 


\subsection{Prediction Results}

For out-of-sample prediction, we randomly choose 100 individuals for the prediction sample and distribute the remaining individuals as estimation samples. In the $5,400(=100 \times T$ and $T=54)$ points of the prediction sample, 704 observations are viewers and 4,696 observations are non-viewers.

Table 5 shows the prediction results, where the numbering for models (1) to (5) is the same as that in the previous section for the estimation results. We utilize three methods to report the prediction results. First, we show the mean squared errors (MSE) between the realized behavior $y$ and predicted choice probabilities for viewing dramas in column (B). Second, we consider group-mean choice probabilities for those who actually viewed a drama in column (C) and those who did not in column (D). For a good predictor, we expect there to be a positive difference between the choice probabilities of the viewed and non-viewed groups.

Third, we report the rate of correct prediction. For the prediction on $t$ th drama in one-step ahead prediction, the rate is defined as

$$
\begin{aligned}
& \frac{1}{N}\left[\sum_{i: y_{i t}=1} \operatorname{Pr}\left(\widehat{y_{i t}}=1\right)+\sum_{i: y_{i t}=0} \operatorname{Pr}\left(\widehat{y_{i t}}=0\right)\right] \\
& =\frac{1}{N}\left\{N_{1}\left[\frac{\sum_{i: y_{i t}=1} \operatorname{Pr}\left(\widehat{y_{i t}=1}\right)}{N_{1}}\right]+\left(N-N_{1}\right)\left[\frac{\sum_{i: y_{i t}=0} \operatorname{Pr}\left(\widehat{y_{i t}=0}\right)}{N-N_{1}}\right]\right\},
\end{aligned}
$$

where $N_{1}$ is the number of those who with $y_{i t}=1$. Because columns A, C and $\mathrm{D}$ in Table 5 correspond to $N_{1} / N, \quad \sum_{i: y_{i t}=1} \operatorname{Pr}\left(\widehat{y_{i t}}=1\right) / N_{1}$ and $1-\left[\sum_{i: y_{i t}=0} \operatorname{Pr}\left(\widehat{y_{i t}=0}\right)\right] /\left(N-N_{1}\right)$, the rate can be represented as $\mathrm{A} \times \mathrm{C}+$ $(1-A) \times(1-D)$. For out-of-sample prediction, the rate is defined as

$$
\frac{1}{N T}\left[\sum_{(i, t): y_{i t}=1} \operatorname{Pr}\left(\widehat{y_{i t}}=1\right)+\sum_{(i, t): y_{i t}=0} \operatorname{Pr}\left(\widehat{y_{i t}=}\right)\right],
$$

and the same representation using columns in Table 5 holds. If one utilizes a random prediction scheme where $\operatorname{Pr}\left(\widehat{y_{i t}=} 0\right)=\operatorname{Pr}\left(\widehat{y_{i t}}=1\right)=0.5$ for any $i$ and $t$, this rate becomes $\left[0.5 N_{1}+0.5\left(N-N_{1}\right)\right] / N=0.5$. Thus, if the rate is larger than 0.5 , it shows that the corresponding prediction scheme yields better performance than the random prediction.

For one-step-ahead prediction, the most important finding of our study is that the CBDT models generally outperform the EUT ones. For the MSE, the EUT models show larger values except for model (4) with respect to the 52nd drama than any case-based models specification in all dramas. 
Table 5: Prediction results.

\begin{tabular}{|c|c|c|c|c|c|c|c|}
\hline & \multirow[t]{2}{*}{$\begin{array}{r}\text { (A) Average } \\
\text { of } y\end{array}$} & \multirow[t]{2}{*}{ (B) MSE } & \multicolumn{4}{|c|}{$\begin{array}{r}\text { Mean of predicted } \\
\text { choice probabilities }\end{array}$} & \multirow{2}{*}{$\begin{array}{r}\text { (E) Rate of } \\
\text { correct } \\
\text { prediction }\end{array}$} \\
\hline & & & & (C) $y=1$ & & (D) $y=0$ & \\
\hline \multicolumn{8}{|c|}{ One-step ahead } \\
\hline$T_{e}+1=51$ & 0.267 & & & & & & \\
\hline (1) & & 0.149 & 0.364 & $(0.232)$ & 0.156 & (0.109) & 0.716 \\
\hline (2) & & 0.152 & 0.364 & $(0.252)$ & 0.158 & $(0.112)$ & 0.714 \\
\hline (3) & & 0.217 & 0.123 & (0.039) & 0.114 & $(0.400)$ & 0.682 \\
\hline (4) & & 0.190 & 0.207 & $(0.170)$ & 0.096 & $(0.104)$ & 0.718 \\
\hline (5) & & 0.199 & 0.207 & $(0.056)$ & 0.126 & $(0.089)$ & 0.696 \\
\hline$T_{e}+1=52$ & 0.227 & & & & & & \\
\hline (1) & & 0.162 & 0.820 & $(0.251)$ & 0.320 & $(0.283)$ & 0.712 \\
\hline (2) & & 0.162 & 0.816 & $(0.252)$ & 0.319 & $(0.282)$ & 0.712 \\
\hline (3) & & 0.199 & 0.371 & $(0.068)$ & 0.367 & $(0.067)$ & 0.573 \\
\hline (4) & & 0.158 & 0.597 & $(0.222)$ & 0.345 & $(0.155)$ & 0.642 \\
\hline (5) & & 0.204 & 0.504 & $(0.080)$ & 0.398 & $(0.179)$ & 0.580 \\
\hline$T_{e}+1=53$ & 0.253 & & & & & & \\
\hline (1) & & 0.164 & 0.619 & $(0.281)$ & 0.291 & $(0.244)$ & 0.687 \\
\hline (2) & & 0.165 & 0.611 & $(0.284)$ & 0.288 & $(0.245)$ & 0.687 \\
\hline (3) & & 0.188 & 0.254 & $(0.068)$ & 0.239 & $(0.063)$ & 0.633 \\
\hline (4) & & 0.188 & 0.364 & $(0.216)$ & 0.219 & $(0.149)$ & 0.676 \\
\hline (5) & & 0.166 & 0.363 & $(0.096)$ & 0.261 & $(0.145)$ & 0.644 \\
\hline$T_{e}+1=54$ & 0.439 & & & & & & \\
\hline (1) & & 0.148 & 0.555 & $(0.258)$ & 0.190 & $(0.148)$ & 0.698 \\
\hline (2) & & 0.149 & 0.553 & $(0.262)$ & 0.188 & $(0.145)$ & 0.698 \\
\hline (3) & & 0.278 & 0.256 & $(0.066)$ & 0.237 & $(0.058)$ & 0.540 \\
\hline (4) & & 0.244 & 0.316 & $(0.188)$ & 0.184 & $(0.087)$ & 0.596 \\
\hline (5) & & 0.231 & 0.346 & $(0.086)$ & 0.226 & $(0.145)$ & 0.586 \\
\hline \multicolumn{8}{|c|}{ Out-of-sample } \\
\hline Naive & 0.130 & & & & & & \\
\hline (1) & & 0.097 & 0.311 & $(0.227)$ & 0.155 & $(0.094)$ & 0.775 \\
\hline (2) & & 0.097 & 0.310 & $(0.226)$ & 0.155 & $(0.094)$ & 0.775 \\
\hline (3) & & 0.115 & 0.251 & $(0.084)$ & 0.202 & $(0.076)$ & 0.727 \\
\hline (4) & & 0.100 & 0.318 & $(0.201)$ & 0.162 & $(0.112)$ & 0.770 \\
\hline (5) & & 0.101 & 0.302 & $(0.132)$ & 0.158 & $(0.127)$ & 0.772 \\
\hline Aggregate & 0.130 & & & & & & \\
\hline (1) & & 0.123 & 0.204 & $(0.081)$ & 0.199 & $(0.076)$ & 0.723 \\
\hline (2) & & 0.123 & 0.213 & $(0.099)$ & 0.199 & $(0.086)$ & 0.724 \\
\hline (3) & & 0.115 & 0.251 & $(0.084)$ & 0.202 & $(0.076)$ & 0.727 \\
\hline (4) & & 0.098 & 0.326 & $(0.207)$ & 0.159 & $(0.113)$ & 0.774 \\
\hline (5) & & 0.100 & 0.307 & (0.138) & 0.155 & $(0.129)$ & 0.775 \\
\hline
\end{tabular}

Note: (1) to (5) represent the homogeneous and heterogeneous case-based models and EUT models without a subjective evaluation, with a subjective evaluation for a previous drama and the mean subjective evaluation of previously watched dramas, respectively. Standard deviations are in parentheses. 
For predicted choice probabilities, the EUT models show similar means for those who actually viewed dramas and those who did not, whereas the models indicate a large positive difference in choice probabilities between viewed and non-viewed groups. Specifically, probability differences are between $20 \%$ and $50 \%$ points for the CBDT models. On the other hand, the differences are $1 \%$ to $2 \%$ points for the EUT model without subjective evaluation while they are $10 \%$ to $15 \%$ points for the EUT model with subjective evaluations. These results are consistent with our finding on the superiority of the CBDT models in statistical model selection. Among the EUT models, the incorporation of subjective evaluation increases the prediction performance, which is also consistent with the model selection result.

In the rates of correct prediction, the CBDT models generally show better values than the EUT models, with one exception of for model (4) with respect to the 51st drama. The CBDT models achieve about $70 \%$ of rates of correct prediction, which shows the moderate prediction power of our methodology.

For one-step-ahead prediction among the CBDT models, the homogeneous model achieved smaller MSEs for three dramas, $T_{e}+1=51,53,54$, and a similar MSE for $T_{e}+1=52$. Thus, the homogeneous model has better prediction performance than the heterogeneous model does. This result shows the minor role of demographic information in the CBDT model given subjective evaluations, which is also consistent with the implications of the estimation results.

For naive out-of-sample prediction, the CBDT models again outperform the EUT ones. However, the EUT models with subjective evaluations achieve similar performance to the CBDT models. This might imply that the EUT models with subjective evaluations are robust for out-of-sample prediction analysis because they do not utilize individual variations of subjective evaluations for estimation.

The question remains as to whether we can mimic individual-level information with aggregate-level information for the CBDT model. In out-of-sample prediction with aggregate data, case-based models show such poor performance that they are outperformed by the EUT models, in contrast to the previous results. Furthermore, the EUT models show smaller prediction MSEs, and the CBDT models do not show a large difference in the mean choice probabilities between viewed and not-viewed samples. Specifically, the probability differences are approximately 0.5 or $1.5 \%$ points for the CBDT models, while the differences are about $15 \%$ points for the EUT model with subjective evaluation. Even the EUT model without subjective evaluation can achieve a $5 \%$ points difference, which outperforms the CBDT model.

These results imply that we cannot replace the individual subjective evaluations by demographic information and aggregate data for the CBDT model. 
Therefore, the good performance of the CBDT model in statistical model selection and one-step-ahead prediction might depend on the availability of individual subjective evaluations, which imposes a burden for data collection in practice.

It is also interesting to consider whether our approach can show superior prediction performance to a simple prediction scheme that $y_{i t}=0$ for any $i$ and $t$. Our prediction analysis so far is mainly based on the predicted choice probabilities. On the other hand, as suggested by Cameron and Trivedi (2005, 474), prediction for binary variables has a different approach which constructs a predictor for the binary dependent variable itself. Because the simple prediction scheme belongs to the latter approach, we compare the performance between the binary predictors from our methodology and from the simple prediction. For a predictor from our methodology, we utilize a scheme where $y_{i t}=1$ if the corresponding predicted choice probability exceeds or is equal to 0.5. We abbreviate to show detailed results to save space, but our CBDT models outperforms the simple prediction for all analysis in Table 5, except for the out-ofsample prediction with aggregate data which is already shown as a difficult target for the CBDT.

\subsection{Extensions}

In this subsection, we utilize two extensions to our basic model, which are possible owing to the flexibility of our linear probit model. Specifically, we consider a premium for recently viewed dramas and include the published audience rate as an explanatory variable. We introduce the following extensions into the homogeneous CBDT model in which $w_{i}=w .^{9}$

First, we introduce a special treatment for recently viewed dramas. It is natural that audiences are more affected by recent dramas, which are fresh in their memories. As mentioned in Section 3.1, our CBDT model so far assumes that individuals employ time-invariant decision making. In contrast, our analysis in this extension adopts time-dependent similarities into the CBDT model.

In this study, we multiply the similarity function by an additional variable $\alpha>0$ when the $\tau$-th drama was broadcast within the previous year. Because there are four getsuku dramas per year, dramas in the previous year are identified by $t-\tau \leq 4$. Thus, our new similarity function becomes

9 We also introduced these extensions into the heterogeneous case-based model. Because the results were similar to those for the homogeneous model, we omit reporting them here. 


$$
s_{i}\left(\mathbf{x}_{t}^{P}, \mathbf{x}_{\tau}^{P}\right)=\alpha^{I[t-\tau \leq 4]} \sum_{k} \omega_{i k} I\left[\mathbf{x}_{k t}^{P}=\mathbf{x}_{k \tau}^{P}=1\right] .
$$

In this new model, $\alpha=1$ means that recent dramas have the same effects as less-recent programs, whereas $\alpha>1$ means that recent programs are more influential.

Second, we adopt the audience rate of dramas to control two elements. The first element is peer effects. As mentioned in Gilboa and Schmeidler (2001, 34), the CBDT model is originally aimed at decision-making using not only own previous experience but also by other decision-makers. This notion is commonly seen in cultural economics as social learning, such as the empirical study of movie ticket sales by Moretti (2011). We eliminate the peer effects from family members analyzed by Yang, Narayan, and Assael (2006) by restricting our sample to single households. However, more general peer effects, such as those by colleagues and neighbors, cannot be avoided in our basic model.

A main reason to include the audience rate is to capture such peer influence. Peer effects might be associated with person-to-person communication after the start of broadcasting. In our definition of the variable, the drama viewing dummy takes a value of 1 if an individual viewed a drama at least once. This definition can capture an audience who starts viewing a drama from the second or later episode as a result of peer effects. We interpret that this specification with audience rate implies a hybrid model of learning from own experience and social learning from peers ${ }^{10}$ in the CBDT model. There can be interaction between these two learning mechanisms, but detailed consideration of such theoretical study is beyond the scope of this article.

The audience rate can also control impacts from other TV programs in the 9 pm Monday timeslot. Competition between TV networks for timeslots may exist, like that analyzed in Goettler and Shachar (2001). The existence of a strong rival can be partially captured by a low audience rate.

Table 6 reports the estimation and prediction results for the extended models. (6) shows the results for the model with the premium for recent programs, whereas (7) shows the results for the model with the published audience rate. For estimation results, (6) shows that the estimated value of $\alpha$ is significantly positive. Furthermore, the one-sided test for the hypothesis of $\alpha>1$ is not rejected at the 0.01 significance level. This result implies the existence of a strong premium for recently viewed dramas in the CBDT model. In the case of (7), the estimated coefficient for the published audience rate is shown to be

10 Kinjo and Ebina (2015) estimated a hybrid model containing a state-dependent term and audience rate under a similar motivation to that in this article. 
Table 6: Results for extended models.

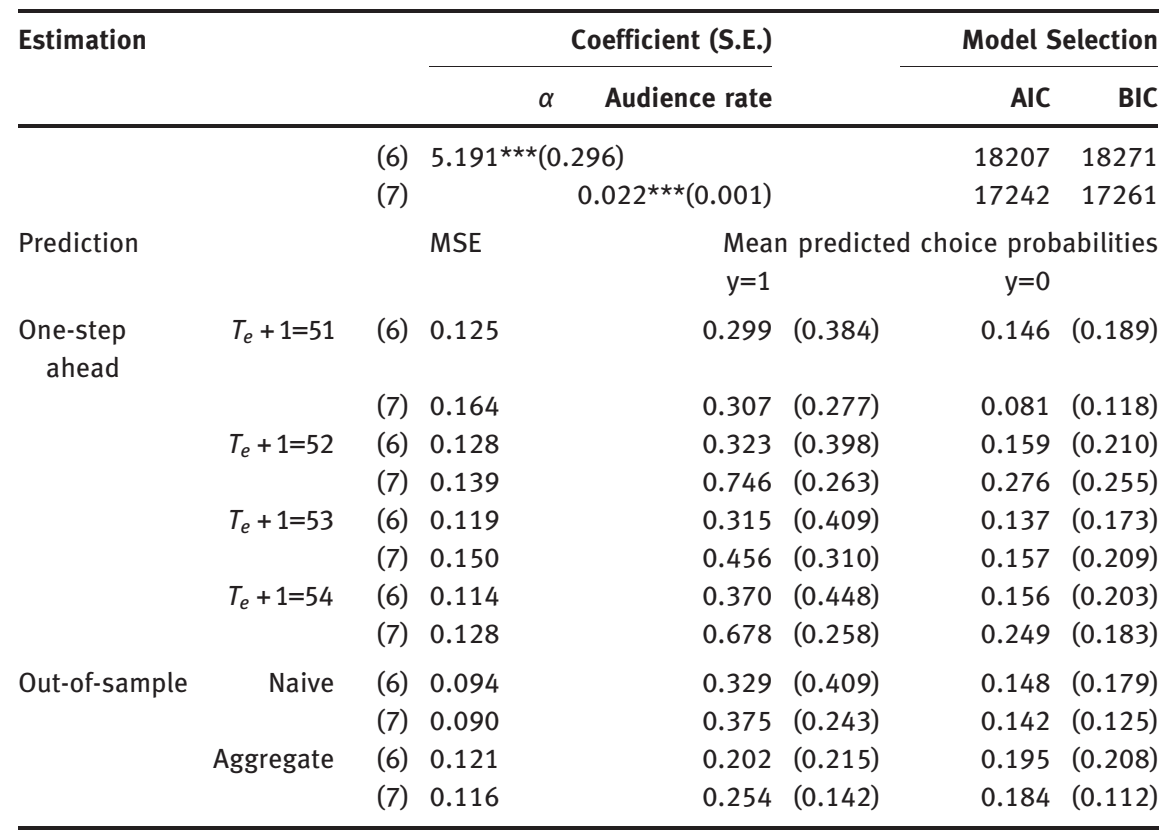

Note: (6) and (7) represent models with a premium for recently viewed dramas and with published audience rate, respectively. The coefficient estimate for (7) shows the marginal effect of probit estimation. Standard errors for estimation and standard deviations for prediction are in parentheses. ${ }^{* \star \star}$ in estimation denotes $p<0.01$.

significantly positive. This result is naturally interpreted as either a positive peer effect or the existence of a premium from the absence of a strong rival.

For the model performance, we show that both extended models outperform the basic CBDT model in Tables 2 and 5. AIC, BIC, and one-step-ahead and naive out-of-sample prediction results show better performance of extended models than basic models for the CBDT and the EUT. On the other hand, the out-ofsample prediction with aggregate information performs better than the basic CBDT models but worse than the EUT models. These results indicate the importance of the extended models in the empirical analysis as an extension of CBDT, but the models cannot substitute EUT when we have access only to aggregate information for subjective evaluations.

A reservation must be made in interpreting the result in (7), because the inclusion of the audience rate as an explanatory variable for the viewing decision can entail the reflection problem of Manski (1993). The reflection problem is an identification problem where an explanatory variable is a function 
of a dependent variable. For our situation, a justification is that the probability of overlapping samples for the research of the published audience rate and our web survey are extremely low and can be negligible. Thus, the published audience rate is not directly related to viewing behaviors of our sample. However, because these two samples are both aimed at random sampling from the same population, there may still be endogeneity caused by unobservable elements. Because it is difficult to obtain a valid instrument, such an endogeneity problem remains a potential problem of our estimation in (7).

\section{Conclusion}

This study empirically analyzed audience behavior for Japanese TV viewing using the CBDT model. We constructed a simple linear probit model and proposed prediction methods for audience rates. Our empirical analysis showed the superior performance of our model in comparison with a conventional EUT model. The CBDT model is revealed to be such a powerful model for TV viewing behavior that it can work well even without consumer characteristics other than individual subjective evaluations.

There are two remaining concerns for empirical applications of the CBDT model. The first concern is estimation of the aspiration level. We avoid direct estimation of this variable, because estimation for it requires an advanced econometric methodology or non-trivial assumptions regarding the adjustment process. The second concern is about our empirical strategy of ignoring consumer behaviors before the research period. Such data trimming might be practically inevitable for empirical application of the CBDT model, but biased estimation may occur. On the other hand, our analysis in Section 4.3 shows that recently watched programs have more importance in the formulation of the similarity function. Then, past drama effects may decay over time, which justifies the data trimming. Because these concerns need to overcome not only empirical but also theoretical difficulties, we believe that further studies involving collaboration between theoretical and empirical researchers can extend the CBDT frontier.

A potential weakness of this study is the small sample size in both the number of individuals and the coverage of TV programs. For individuals, the limited sample size restricts the number of discretized categories for demographic characteristics, despite that we originally have finer information. On the coverage of programs, although our research concentrated on getsuku dramas, influence by other drama programs may occur. This is indicated by 
the fact that no actress appeared in more than three getsuku dramas as a main character. It would not be natural that drama producers ignore the influence of actresses on TV viewing behavior. Thus, this observation might imply that the popularity of actresses, at least in youth-oriented dramas such as getsuku, vanishes quickly. Thus, it might be important to consider the many drama programs broadcast at the same time as getsuku.

Acknowledgments: The authors are grateful to two anonymous referees, Atsuyuki Kogure, Kensuke Miyazawa, Jiro Nakamura, Nobuhiko Terui, and the seminar and workshop participants at Keio University in Tokyo on March 16th, 2013 and JJSS for their helpful discussions and comments. This study is supported by a Grant-in-Aid for Young Scientists (B) No. 25780272 and No. 15K17070 and a Grant-in-Aid for Research Activity Start-up No. 25885021 from the Japanese Ministry of Education, Science, Sports, Culture, and Technology and JST CREST. The computational results are obtained using Stata 13 and Ox version 7 (Doornik, 2012). For the dataset used in this article, the web survey was conducted through Macromill Inc. Because the copyright for the web survey data belongs to the authors, this dataset is available upon request. For the published audience rate, we utilize the information under the permission of Video Research Ltd. and do not have the rights to release the dataset.

Funding: Japanese Ministry of Education, Science, Sports, Culture and Technology (Grant/Award Number: 'Grant-in-Aid for Young Scientist (B) No. 25780272', 'Grant-in-Aid for Young Scientist (B) No. 15K17070', 'Research Activity start-up No. 25885021') JST CREST.

\section{References}

Allenby, G. M., and P. E. Rossi. 1998. "Marketing Models of Consumer Heterogeneity." Journal of Econometrics 89:57-78.

Becker, G. S., and K. M. Murphy. 1988. "A Theory of Rational Addiction.” Journal of Political Economy 96:675-700.

Bethlehem, J. 2010. "Selection Bias in Web Surveys." International Statistical Review 78:161-88.

Brito, P., and C. Barros. 2005. "Learning-by-Consuming and the Dynamics of the Demand and Prices of Cultural Goods." Journal of Cultural Economics 29:83-106.

Burnham, K. P., and D. R. Anderson. 2002. Model Selection and Multimodel Inference: A Practical Information-Theoretic Approach, 2nd ed. New York: Springer.

Camera, G., and M. Casari. 2009. "Cooperation among Strangers under the Shadow of the Future.” American Economic Review 99:979-1005. 
Cameron, C., and P. K. Trivedi. 2005. Microeconometrics: Methods and Applications. New York, NY: Cambridge University Press.

Danaher, P. J., T. Dagger, and M. Smith. 2011. "Forecasting Television Ratings.” International Journal of Forecasting 27:1215-40.

Doornik, J. A. 2012. Object-Oriented Matrix Programming Using Ox. London: Timberlake Consultants Press and Oxford.

Fujihira, Y. 2007. The Right Way to Use Audience Rate (Shichouritsu No Tadashii Tsukaikata, in Japanese). Tokyo, Japan: Asahi Shinbunsha.

Gayer, G., I. Gilboa, and O. Lieberman. 2007. "Rule-Based and Case-Based Reasoning in Housing Prices." The B.E. Journal of Theoretical Economics 7.

Gilboa, I., O. Lieberman, and D. Schmeidler. 2006. "Empirical Similarity." Review of Economics and Statistics 88:433-44.

Gilboa, I., O. Lieberman, and D. Schmeidler. 2011. "A Similarity-Based Approach to Prediction." Journal of Econometrics 162:124-31.

Gilboa, I., and D. Schemeidler. 1995. "Case-Based Decision Theory.” Quarterly Journal of Economics 110:605-39.

Gilboa, I., and D. Schmeidler. 1996. "Case-Based Optimization.” Games and Economic Behavior 15:1-26.

Gilboa, I., and D. Schmeidler. 2001. A Theory of Case-Based Decisions. Cambridge, MA: Cambridge University Press.

Goettler, R. L., and R. Shachar. 2001. "Spatial Competition in the Network Television Industry." RAND Journal of Economics 32:624-56.

Golosnoy, V., and Y. Okhrin. 2008. "General Uncertainty in Portfolio Selection: A Case-Based Decision Approach.” Journal of Economic Behavior and Organization 67:718-34.

Guilfoos, T., and A. D. Pape. 2016. "Predicting Human Cooperation in the Prisoner's Dilemma Using Case-Based Decision Theory." Theory and Decision, 80:1-32.

Higashino, K. 2012. The Devotion of Suspect X: A Detective Galileo Novel. New York, NY: Minotaur Books, English translation.

Ichimura, H., and T. S. Thompson. 1998. "Maximum Likelihood Estimation of a Binary Choice Model with Random Coefficients of Unknown Distribution." Journal of Econometrics 86:269-95.

Iwabuchi, K. ed. 2004. Feeling Asian Modernities: Transnational Consumption of Japanese TV Dramas. Hong Kong: Hong Kong University Press.

Japan Ministry of Public Management, Home Affairs, Posts and Telecommunications. 2012. Communications Usage Trend Survey (Tsuushin Riyou Doukou Chousa, in Japanese). Tokyo.

Kinjo, K., and T. Ebina. 2015. "State-Dependent Choice Model for TV Programs with Externality: Analysis of Viewing Behavior." Journal of Media Economics 26:20-40.

Lévy-Garbova, L., and C. Montmarquette. 1996. "A Microeconomic Study of Theatre Demand.” Journal of Cultural Economics 29:25-50.

Lieberman, 0. 2010. “Asymptotic Theory for Empirical Similarity Models." Econometric Theory 26:1032-59.

Lieberman, O. 2012. "A Similarity-Based Approach to Time-Varying Coeffcient Nonstationary Autoregression." Journal of Time Series Analysis 33:484-502.

Lieberman, O., and P. C. B. Phillips. 2014. "Norming Rates and Limit Theory for Some Time-Varying Coefficient Autoregressions." Journal of Time Series Analysis 35:592-623. 
Manski, C. 1993. "Identification of Endogenous Social Effects: The Reflection Problem.” Review of Economic Studies 60:531-42.

Matsui, A. 2000. "Expected Utility and Case-Based Reasoning." Mathematical Social Science 39:1-12.

McFadden, D. 1974. "Conditional Logit Analysis of Qualitative Choice Behavior." In Frontiers in Econometrics, edited by P. Zarembka, 105-42. New York: Academic Press.

McFadden, D. 1979. "Quantitative Methods for Analyzing Travel Behavior of Individuals: Some Recent Developments." In Behavioral Travel Modeling, edited by D. Hensher and P. Stopher, 270-318. London: Croom-Heim.

Moretti, E. 2011. "Social Learning and Peer Effects in Consumption: Evidence from Movie Sales." Review of Economic Studies 78:356-93.

Moshkin, N. V., and R. Shachar. 2002. "The Asymmetric Information Model of State Dependence.” Marketing Science 21:435-54.

Ossadnik, W., D. Wilmsmann, and B. Niemann. 2013. "Experimental Evidence on Case-Based Decision Theory." Theory and Decision 75:211-32.

Pape, A. D., and K. J. Kurtz. 2013. "Evaluating Case-Based Decision Theory: Predicting Empirical Patterns of Human Classification Learning." Games and Economic Behavior 82:52-65.

Rust, R. T., and M. I. Alpert. 1984. "An Audience Flow Model of Television Viewing Choice." Marketing Science 3:113-24.

Seaman, B. A. 2006. "Empirical Studies of Demand for the Performing Arts." In Handbook of the Economics of Art and Culture 1, edited by V. A. Ginsburgh and D. Throsby, 415-572. Amsterdam: Elsevier.

Shachar, R., and J. W. Emerson. 2000. "Cast Demographics, Unobserved Segments, and Heterogeneous Switching Costs in a Television Viewing Choice Model.” Journal of Marketing Research 37:173-86.

Teitelbaum, J. C. 2015. “Analogical Legal Reasoning: Theory and Evidence.” American Law and Economics Review 17:160-91.

Yang, S., V. Narayan, and H. Assael. 2006. "Estimating the Interdependence of Television Program Viewership between Spouses: A Bayesian Simultaneous Equation Model.” Marketing Science 25:336-49. 